\title{
Production, Measurement and Applications of Vacuum Systems
}

\author{
Shailaj Kumar Shrivastava, Chandan Shrivastava
}

\begin{abstract}
The most common type of vacuum pumps and measuring gauges based on available literature are studied with emphasis on how new research and development will enable the new generation of vacuum technology specially in designing, its operational procedure and applications. The technologies were developed to meet the operational goal which include vacuum chamber structures, compatible materials, specialized vacuum pump and gauges. There are many areas where different vacuum condition is required for conducting experiments therefore modeling of pumping system is on demand. The basic understanding of how and when the particular pumping and measurement system can be applied most effectively and economically is essential. The poor choice of pumping and measurement system will interfere the scientific objectives and may leads to substantial maintenance demands and an unpleasant working environment. The development and fundamental investigation of innovative vacuum techniques for creation and measurement of vacuum used for various applications necessary for the research work to be done in future are presented.
\end{abstract}

Keywords: Positive displacement vacuum pump, Momentum transfer vacuum pump, Entrapment vacuum pump, Thermal conductivity gauge, Ionization gauge, leak detection.

\section{INTRODUCTION}

A high or ultrahigh vacuum with measuring system is essential for scientific experiments and for the development of solid-state microelectronics and micromechanical devices. A vacuum pump and measuring gauge are the most essential component of any vacuum system, therefore to understand its applications, there is need to give introduction to the concept of creating, measuring and maintaining of vacuum system. The different measuring vacuum levels from atmospheric pressure (760 Torr) to ultra-high vacuum $\left(10^{-9}\right.$ to $10^{-12}$ Torr) are listed in table1. The solution of many complex scientific and technological problems requires the attainment of pressures of the order of $10^{-14} \mathrm{~N} / \mathrm{m}^{2}$ and lower, as well as the measurement of such pressures. However, pressures as low as $5 \times 10^{-17}$ Torr corresponding to $\approx 100$ particles $/ \mathrm{cm}^{3}$ have been indirectly measured in a $4 \mathrm{~K}$ cryogenic vacuum system.

Manuscript received on February 17, 2021.

Revised Manuscript received on February 20, 2021.

Manuscript published on February 28, 2021

*Corresponding author:

Shailaj Kumar Shrivastava*, Principal, Daudnagar College (A constituent unit of Magadh university, Bodh Gaya), Aurangabad, Bihar, 824113, India. Email: shailajshri68@yahoo.com

Chandan Shrivastava, Undergraduate student, Computer Science Engineering (CSE), International Institute of Information Technology, Hyderabad, Telangana, 500032, India. E-mail: chandanshrivastava2002@gmail.com

(C) The Authors. Published by Blue Eyes Intelligence Engineering and Sciences Publication (BEIESP). This is an open access article under the CC BY-NC-ND license (http://creativecommons.org/licenses/by-nc-nd/4.0/)
Table 1. Different vacuum levels

\begin{tabular}{|l|l|}
\hline Atmospheric pressure & 760Torr \\
\hline Low Vacuum & 760 Torr to 25 Torr \\
\hline Medium Vacuum & 25 Torr to $1 \times 10^{-3}$ Torr \\
\hline High Vacuum & $1 \times 10^{-3}$ Torr to $1 \times 10^{-9}$ Torr \\
\hline Ultra-High Vacuum & $1 \times 10^{-9}$ Torr to $1 \times 10^{-12}$ Torr \\
\hline $\begin{array}{l}\text { Extremely High } \\
\text { Vacuum }\end{array}$ & $<1 \times 10^{-12}$ Torr \\
\hline Outer Space & $<1 \times 10^{-17}$ Torr \\
\hline Perfect Vacuum & 0 Torr \\
\hline
\end{tabular}

The direct gauges measure the actual vacuum pressure which include the Bourdon and capacitance manometer. But it is difficult to measure below $10^{-5}$ Torr by direct measurement. The indirect pressure gauge calculates the vacuum pressure by measuring a physical property of the gas which changes in a predictable manner as gas density changes so it must be calibrated for the gas type used. Therefore, the choice of suitable gauge depends on the working principle of the gauge, the pressure range and its accuracy. A highly accurate ionization gauge in the range $10^{-3}-10^{-8}$ Torr will need an accurate emission current control and accurate ion current measurement. For extremely high vacuum $\left(10^{-12}-10^{-15}\right.$ Torr $)$ region monopole and quadrupole mass spectrometers are used. The vacuum gauges and their measuring ranges are shown in table 2.

Table 2. Vacuum gauges and their range

\begin{tabular}{|l|l|}
\hline Bourdon gauge & $10^{3}$ Torr to 1 Torr \\
\hline Capacitance Manometer & $1 \times 10^{3}$ Torr to $1 \times 10^{-5}$ Torr \\
\hline Mc Leod Gauge & 10 Torr to $1 \times 10^{-6}$ Torr \\
\hline $\begin{array}{l}\text { Pirani Gauge and } \\
\text { thermocouple gauge }\end{array}$ & 10 Torr to $1 \times 10^{-4}$ Torr \\
\hline $\begin{array}{l}\text { Hot Cathode ionization } \\
\text { Gauge }\end{array}$ & $\begin{array}{l}1 \times 10^{-3} \text { Torr to } 1 \times 10^{-11} \\
\text { Torr }\end{array}$ \\
\hline Cold Cathode Gauge & $\begin{array}{l}1 \times 10^{-3} \text { Torr to } 1 \times 10^{-13} \\
\text { Torr }\end{array}$ \\
\hline
\end{tabular}

The vacuum pumps are used for a wide range of applications in freeze drying of foods and pharmaceuticals $\left(10^{-2}-10^{-3}\right.$ Torr $)$, vacuum coating of metals, nuclear particle accelerators $\left(10^{-5}-10^{-14}\right.$ Torr $)$, thin film processing $\left(10^{-1}-10^{-8}\right.$ Torr), semiconductor processing $\left(10^{-6}-10^{-9}\right.$ Torr $)$, electron beam welding, surface studies $\left(10^{-9}{ }_{-} \quad 10^{-14}\right.$ Torr $)$ and multifunctional medical central vacuum system. If the vacuum pump is operated under the condition which may cause unappreciated impact on pressure gauge reading, extra care will be taken for its cleanliness, safety and maintenance. The pumps and their ranges are listed 3.

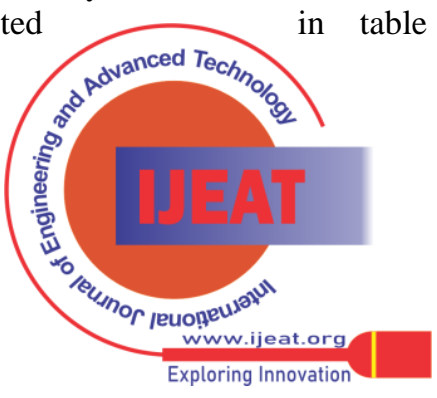

Blue Eyes Intelligence Engineering and Sciences Publication 


\section{Production, Measurement and Applications of Vacuum Systems}

Table 3. The pumps and their ranges

\begin{tabular}{|l|l|}
\hline Pumps displacement gas & Ranges \\
\hline $\begin{array}{l}\text { Positive } \\
\text { transfer vacuum pumps }\end{array}$ & $10^{-3}$ Torr \\
\hline $\begin{array}{l}\text { Momentum transfer kinetic } \\
\text { vacuum pumps }\end{array}$ & $10^{-3}-10^{-7}$ Torr \\
\hline Entrapment vacuum pumps & $10^{-4}-10^{-12}$ Torr \\
\hline
\end{tabular}

\section{VACUUM PUMPS}

The principal parameters of vacuum pumps are the ultimate pressure that can be attained by the pump, the evacuation rate and the permissible outlet pressure in the discharge section of the pump, if exceeded, would disrupt the normal operation of the pump.

\section{A. Positive displacement gas transfer vacuum pump}

\section{Rotatory vane vacuum pump}

A rotary vane vacuum pump is an oil sealed rotatory positive displacement mechanical pump. It consists of a stationary part cylindrical stator and eccentrically installed moving part, rotor, assembled inside a protected casing. Spring loaded sliding vanes are mounted in the slots of the rotor that moves radially under spring force are in continuous contact with the stator walls. Gas from the vessel is allowed to enter a region of the pump through the pump inlet port and are trapped. The region is then sealed, the gas inside is compressed resulting in decrease of volume between the rotor and stator and then spring-loaded exhaust valves are used to expel this compressed gas. This valve operated only at a preset pressure to avoid the back flow. is expelled to the atmosphere. The oil sealed rotary pump is shown in figure 1 . Perfect sealing is maintained between the rotor and vanes and duo seal between vanes and the stator chambers by oil. Rotary vane pumps are not very suitable for pumping corrosive gases and vapors, since they contaminate the oil. So, suitable protection, such as cold trap should therefore always be used. Two stages rotatory vane vacuum pumps achieve lower ultimate pressures than single stage pumps. The drawback of rotary vacuum pump is overcome by the development of guarded vane rotary vacuum pump [1]. The operating stability and efficiency of a guarded vane vacuum pump is increased by reducing the rate of depreciation of the basic working surfaces and minimizing losses due to wear of the parts of the pumps. As a result of wear in inner surface of the rotor slots, the outer and lateral surfaces of the working plates, and the inner surfaces of the pump casing the growth of leakage of gas may takes place. Therefore, during operation oil layer shift at the site near rotor and casing. The insulation is maintained by using wear resistive materials with hardness less than that of inner surface of pump casing.

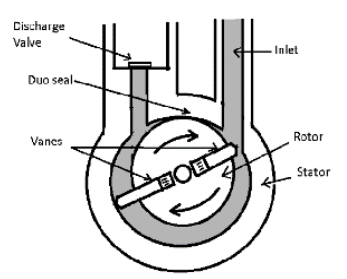

Figure 1. Oil sealed rotary pump
The guarded vane vacuum pump is suitable because of its high discharge rate, design simplicity, lower dimension and weight. It is important to accurately measure, evaluate and predict the chamber pressure variation and the flow rate characteristics at inlet and outlet. Therefore, a numerical model/method, for example Computer Fluid Dynamics (CFD), is needed to understand the flow characteristics of the pump associated with vane rotation and eccentric rotor as well as the two-phase flow of air and lubrication oil between moving and stationary parts [2],[3],[4]. The performance of pump has been affected by the check valve and groove. A check valve at the inlet prevents the reverse movement of air when the rotor rotates in opposite direction. The other check valve attached to the outlet found to significantly reduce the torque of the vacuum pump as well as prevent back flow from the outlet. The pressure of the chamber depends on the check valve thickness. As its thickness is decreased the time required to attain target pressure is reduced. The numerical results indicated that the inclusion of grooves near the inlet can significantly improve the pump performance. As the vane angle increases from $0^{\circ}$, the vane begins to block the inlet area causing a decrease in the inlet flow rate. A groove included near the inlet area in the pump to reduce the blockage of vane and improves the pump performance. The groove caused a significant increase in the flow rate while vane passed over the inlet. The oil fills in the vane housing and rotor housing minimum gaps and prevents leakage or backflow between the chambers. The vane vacuum pump did not work without lubrication oil as it is essential in pumping the air flow. The range of oil sealed rotary pump is from atmospheric pressure to $10^{-3}$ Torr.

\section{Roots vacuum pump}

The roots vacuum pump is rotary volumetric vacuum pump which is applicable where there is high mass flow rates. It consists of two identical lobed rotors mounted inside a casing do not actually touch each other. These lobed rotors are synchronized by an external precision gear mechanism and are connected to an electric drive. The roots vacuum pump is shown in figure 2. A fine mechanical clearance of $0.3 \mathrm{~mm}$ is maintained between the moving lobes and the stator. As a result, these pumps guarantee friction free movement and can be operated at a very high speed ( 3500 to 4200rpm). The lobes are rotated in opposite direction with respect to each other. The overheating of casing results in thermal expansion of label rotors and thereby, the possible contact between the moving parts. A typical ultimate pressure of $<10^{-3}$ Torr can be achieved.

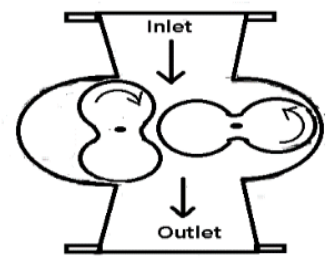

Figure 2 Roots vacuum pumps

Published By:

Blue Eyes Intelligence Engineering and Sciences Publication

156 (c) Copyright: All rights reserved.

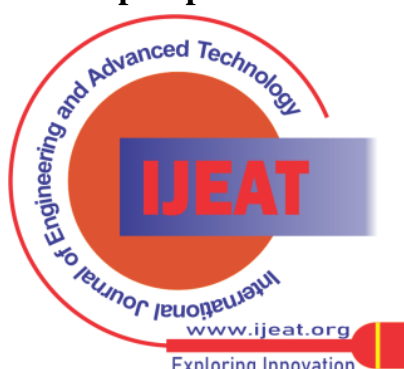




\section{B. Momentum transfers kinetic vacuum pump}

\section{Vapor diffusion pumps}

The oil-diffusion vacuum pump consists of three varying sized, cone shaped pressure jets stacked vertically in one casing. The lowest stacked cone is the largest and decreases in size as moving upwards. A three-stage oil-diffusion pump is shown in figure 3 . In practical application diffusion pump is coupled with a backing pump. The very bottom of the chamber is heater which vaporizes the oil and these hot vapors travel upward into the vapor chimney. The temperature of the oil in vapor state is approximately $180-270^{\circ} \mathrm{C}$. The hot vapors are deflected downwards by an annular nozzle or a jet assembly mounted at the top of the chimney. This jet moving downwards at supersonic speeds, imparts momentum to randomly moving gas molecules in the chamber. This momentum deflects the molecules towards the pump outlet and trap gas molecules along the way through diffusion. The hot oil condenses on cold walls and returns to the vessel at bottom. Initially a mechanical roughing pump is used to remove the gas molecule and then the same mechanical pump is used as the backing pump for a diffusion pump. The hydrocarbon oils are most economical and are widely used in diffusion pumps. These oils are subject to oxidation. The silicon oils which is quite resistant to oxidation are useful for obtaining ultra-high vacuum. The diffusion pumps are effective in the range of $10^{-3}$ to $10^{-7}$ Torr.

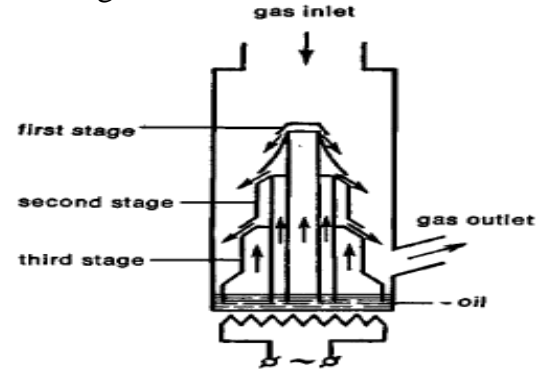

Figure 3. Three-stage oil-diffusion pump

\section{Turbomolecular pump}

Turbomolecular pump consists of alternate layers of stator and rotor discs. The rotor rotates at very high speed. The gas is compressed by several series of rotating blades is followed by a series of static blades. Gas molecules transmitted through the rotating blades, channels hit the static blades so that the angular distribution of velocity is randomized and the molecules are ready for the next compressed stage. The momentum transfer is effective only if the molecules do not experience intermolecular collisions after hitting the blades. Since the mean free path of the gas molecule is greater than the spacing between rotor and stator blades, the molecules that being collide with the blades get adsorbed with rotor blades. Due to continuing full pumping speed, the thermal molecular speed is increased by absorbing momentum from rotor blades. To maximize the pumping speed, the distance between the blades is larger for the first series of blades and their inclination is $45^{\circ}$. A turbomolecular pump is shown in figure 4. The maximum compression ratio is obtained by reducing the distance between the blades and their inclination. The high-speed molecules are directed towards the exit using the fixed stator discs in between rotors. Due to increased momentum molecules get detached from the preceding rotor blades and get attached to the next stator blade. This succession of rotor-stator pairs drives the molecules towards the exhaust where they are collected by backing pump.

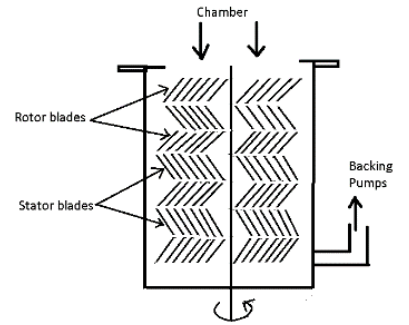

Figure 4 Turbomolecular pump

For stabilization problem in the turbomolecular pumps rotor with significant gyroscopic effects and low resonance frequencies of the blade wheel, active magnetic bearings structure and controller design based on the inverse dynamic system has been developed [5]. With the help of Monte-Carlo simulation the main vacuum characteristics of the multistage turbo molecular pump can be determined after considering the transfer of molecules from one stage to another with some probability, the initial coordinates of the molecule and the direction cosines of the molecular position of the vector which depends on the spatial position of the surface. The MontoCarlo simulation reveals that the performance of the turbomolecular pump depends on the number of blades in a single cross section and geometry of the rotor and stator blades [6]. The pumping speed and other parameters of turbomolecular pumps with multistage flow channel has been obtained which shows its great potential for the effective implementation of task [7]. A turbomolecular pump reduces the pressure of the chamber to about $10^{-8}$ torr.

\section{Entrapment Vacuum pump Sputter ion pump}

In ion pumps the gas is not transferred from vacuum system but is transformed into a condensed phase in the system. Ion pumps are used most efficiently at pressure below $10^{-4}$ Torr. Sputter ion pumps have no moving parts and can provide ultrahigh vacuum pressure as low as $10^{-12}$ Torr. Figure 5 shows ion pump. The ion getter pump consists of an array of positive charged cylindrical anodes and the cathodes are parallel plate of titanium which are kept at negative potential.

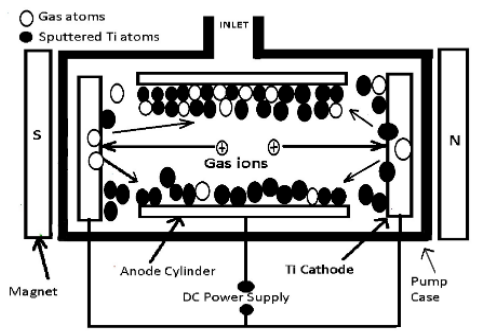

Figure 5. Ion pump

Published By:

Blue Eyes Intelligence Engineering and Sciences Publication

(c) Copyright: All rights reserved.

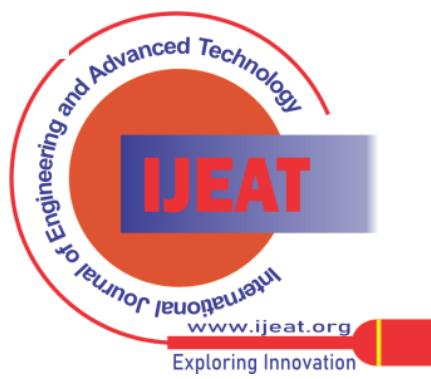




\section{Production, Measurement and Applications of Vacuum Systems}

An aperture anode located between the cathodes. A magnetic field of 0.12 Tesla field strength is applied parallel to the axis of the anode cells through permanent magnets located outside the vacuum chamber. When a potential is applied to the anode, electrons emitted by the cold cathode oscillates back and forth between the cathode in long spiral paths before finally reaching the anode wall. The long path length of the electron results in high probability for collision and ionization between the electron and gas molecules. The cathode must be periodically replaced. The freshly deposited $\mathrm{Ti}$ film gathers the chemically active gases (e.g., $\mathrm{N}_{2}, \mathrm{O}_{2}, \mathrm{H}_{2}, \mathrm{CO}_{2}, \mathrm{CO}$, water vapor and light hydrocarbon) which can be pumped by chemisorption. To achieve the ultimate pressure regime of $10^{-}$

${ }^{11}$ Torr a titanium sublimation pumps are generally used in conjunction with a sputter ion pump, but this combination is not always feasible due to space limitations. The use of compact NEXTorr D2000-10 combination pump provide large speed in a more compact design and is applicable where large load of gases is generated and no more space available [8].

\section{Cryo pump}

Cryo pump is a self-contained, hydrocarbon free and high pumping speed vacuum pump which utilizes extremely low temperatures to condense gases by means of cryocondensation, cryosorption or cryotrapping and thus remove them from the system. A cryopump usually consists of a large number of metal plates and employ helium to cool the low-temperature surface to adsorb gas molecules. Figure 6 shows cryogenic pump. Weak Vander Waals attractive forces may provide molecular gas pumping done in multiple stages where each stage has different temperature. A two stage cold head unit produces temperature of $77 \mathrm{~K}\left(\mathrm{I}^{\mathrm{st}}\right.$ stage $)$ and $20 \mathrm{~K}\left(2^{\mathrm{nd}}\right.$ stage). At temperature of $77 \mathrm{~K}$, the other gas particles cryocondensate along with similar water molecule that have already attached to a cold surface.

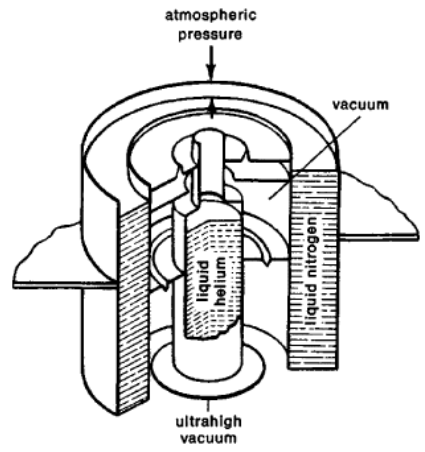

Figure 6. Cryogenic pump

During cryocondensation a non-condensation gas is mixed with a condensation gas may also trapped which in turn is condensed on a cold surface. The interaction energy between dissimilar molecules may be much higher than that between similar molecules therefore the molecules of a low boiling temperature gas are trapped in the condensation layer of another gas. For example, Ar trapped in $\mathrm{CO}_{2}$ at $77 \mathrm{~K}$ and $\mathrm{H}_{2}$ in $\mathrm{N}_{2}$ at 20K. In order to effectively pump gases such as nitrogen less than $20 \mathrm{~K}$ is required. In this stage the metal may also be coated with charcoal that allows to absorb even smaller gas molecules such as helium and even hydrogen. The adsorbent KCC/IIS01 is better for pumping helium gas by cryosorption pump [9].

\section{VACUUM GAUGES}

Different vacuum gauges mechanical, thermal conductivity and ionization gauges based on their methods of operation are required to accurately measure pressure in a vacuum system.

\section{A. Bourdon gauge}

The Bourdon gauge is a direct pressure measurement device that actually measures the force from the vacuum pressure. It consists of a curved hollow tube with elliptical cross-section made of quartz closed at one end and is free to move. The other end of the tube is fixed to the frame. When pressure is less than atmospheric pressure, the free end moves inward resulting in rotation of gauge needle to display the corresponding change in vacuum pressure. The small gears and levers are used to amplify the movement of gauge needle. Bourdon tube gauges are calibrated against known pressure to ensure correct readings.

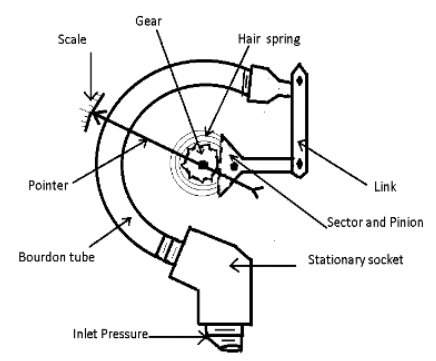

Figure 7 Bourdon tube gauge

Bourdon tubes gauges are suitable for measuring low pressure ranges between atmospheric pressure and 10Torr. Figure 7 shows Bourdon tube gauge. The sensitivity of Bourdon tube depends on its cross-section and material used. The Zinc material and oval cross-section shows better sensitivity [10].

\section{B. Mc-Leod gauge}

The McLeod gauge are the earliest and most well-known forms of manometers for measuring pressures in the low, medium and high vacuum range. These devices measure low gas pressures by compressing a known volume of the gas at constant temperature and is independent of the nature of the gas Figure 8 shows the McLeod gauge. The McLeod gauge takes advantage of Boyle's law to determine gas pressure within a range of 10 to $10^{-6}$ Torr.

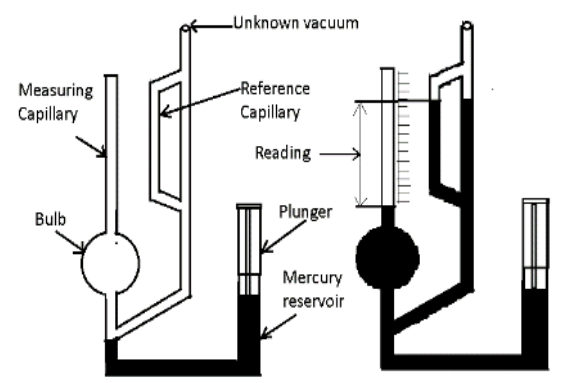

Figure 8. The McLeod gauge

Published By:

Blue Eyes Intelligence Engineering and Sciences Publication

158 (C) Copyright: All rights reserved.

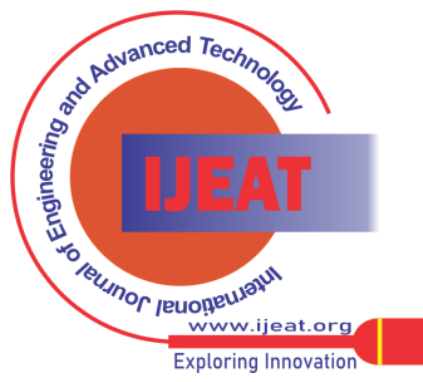


To obtain high accuracy with a McLeod gauge, the capillary should be of uniform bore and glass and mercury must be kept clean and free of water vapor. These gauges are suitable for non-condensable gases like nitrogen and oxygen. But are not suitable for condensable gases like carbon dioxide, ammonia, water or pump oil vapors etc.

\section{Capacitance Manometer Gauge}

The capacitance manometer gauge is a direct pressure measurement electro-mechanical gauge used to measure vacuum from atmospheric pressure to $10^{-5}$ Torr. The gauge contains a small metal diaphragm. Figure 9 shows capacitance manometer gauge. The diaphragm provides very low hysteresis, excellent repeatability, remarkable high resolution, fast response and ability to measure extremely low pressure.

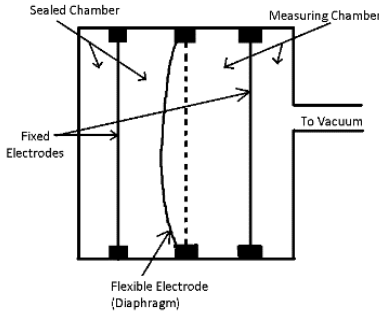

Figure 9. Capacitance Manometer Gauge

A capacitance manometer's signal conditioning electronics will provide an output voltage with a linear relationship between output voltage and pressure. The small gap between the metal diaphragm and the fixed electrode makes this type of gauge especially susceptible to vibration, temperature and any electrical field charges.

\section{Thermal conductivity gauge}

\section{Pirani Gauge}

The pirani gauge is a thermal conductivity gauge used to give a pressure reading due to a heated metal wire suspended in the vacuum system to be measured [11]. As the vacuum is pumped down the gas molecules in the system collide with the wire and wire heats up. Measurement of heat loss is an indirect indication of pressure. When the wire is heated, the electrical resistance increases and the bridge circuit attached to the wire detect the change in resistance.

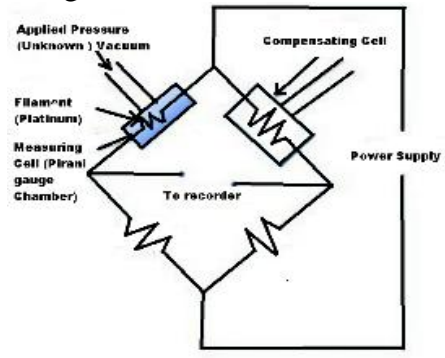

Figure 10 Pirani Gauge

The change in resistance of the pirani gauge filament is proportional to pressure in vacuum becomes a measure of the applied pressure. The pirani gauge is used to measure pressure between 0.5 Torr to $10^{-4}$ Torr. Figure 10 shows pirani gauge. Before using the gauge, the apparatus may need calibrating to obtain accurate reading depending on the thermal conductivity and the heat capacity of the gas.

\section{Thermocouple Gauge}

A thermocouple is a junction between two metals whose work functions are so different that a detectable voltage develops across the junction. For a given amount of current, a thermocouple will get hotter at lower pressures since collisions with gas molecules are less frequent than at higher pressure. Here, a filament is heated by passing a constant current through it. The temperature is directly dependent on the pressure in the chamber.

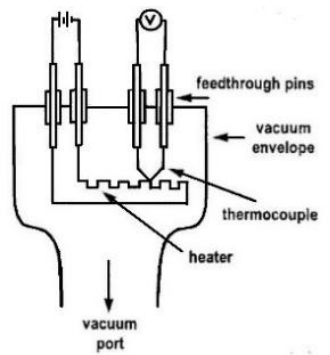

Figure 11 Thermocouple Gauge

The temperature change of the wire is measured precisely by thermocouple, where the generated voltage depend depends on the temperature of the filament. Figure 11 shows thermocouple gauge. The reading of voltmeter calibrated to show pressure. This gauge is used for comparison purposes and the sensitivity varies based on the pressure and the strength of the current. In the thermocouple gauge [12], the hot junction of the thermocouple is attached to a filament in the vacuum system and powered from a constant voltage source. The current through the platinum heater is adjusted to some constant value. The sensitivity of the gauge will change if the surface of the heater becomes contaminated with oxide or absorbed vapor, which changes its thermal emissivity or accommodation coefficient.

\section{E. Ionization gauges}

For the gas pressure in the range $10^{-3}$ to $10^{-11}$ Torr, gas density is extremely low so that direct pressure measurement from vacuum forces is too weak to be measured directly. In this range the vacuum pressure is measured by ionization of the gas. In the high and ultrahigh vacuum range there are two types of ionization gauges hot cathode and cold cathode which sense pressure by detecting ion current resulting from gas ionized by electron bombardments. These two gauges differ in the source of the ionizing electrons, electrode arrangement and dependence of vacuum pressure on ion current.

\section{Cold cathode ionization gauge}

The principle behind cold cathode ion gauge [13] is that electrons are produced in a discharge created by a high voltage electrical discharge. Figure 12 shows cold cathode gauges (Penning gauge). It consists of pair of cathodes placed opposite to one another and a hollow anode in between two cathodes.

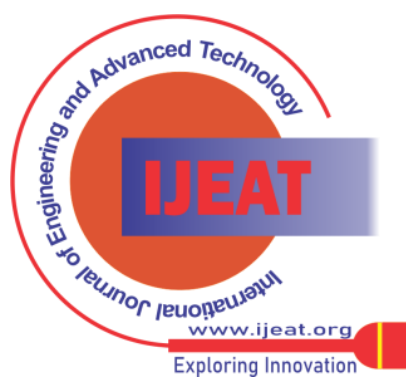




\section{Production, Measurement and Applications of Vacuum Systems}

A permanent magnet provides magnetic fields which increases the path length for the electron in going from anode to cathode, enabling more ionization possible. A high voltage ranges from 2-6 kV and magnetic field of 1-2 kG provides sufficient energy to ionize gas molecules at low pressure of $\sim 10^{-5}$ Torr. The electric current caused by the collection of positive ions created inside the gauge is used as an indirect measure of gas pressure.

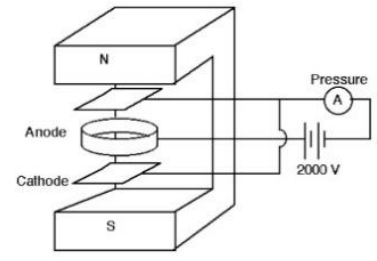

Figure 12. Cold cathode gauges (Penning gauge)

Cold-cathode ionization gauge makes use of the fact that the rate of ion production by a stream of electrons in a vacuum system is dependent on pressure and the ionization probability of the residual gas. This gauge shows negligible thermal outgassing and electron stimulated desorption. Cold cathode ionization gauge measures accurate pressure between $10^{-2}$ and $10^{-7}$ Torr.

\section{Hot cathode ionization gauge}

Hot cathode ionization gauge is composed of three electrodes collector, a filament and a grid [14]. Electrons are thermionic emitted from a hot filament accelerated towards the cylindrical shaped grid and in the meantime, electrons emitted from the filament ionize residual gas molecules in the container being evacuated. The large positive ion thus formed inside the grid are subsequently accelerated and collected by the center ion collector wire held at comparative lower electrical potential. With this arrangement the ion collector intercepts only a small fraction of the X-ray produced at the grid. The ion current flowing through the collector is measured which is proportional to pressure. Figure 13 shows hot cathode ionization gauge. The sensitivity of ionization gauge for a particular gas depends not only on the voltages applied to the gauge and the gauge geometry, but also on the ionization characteristics of the gas.

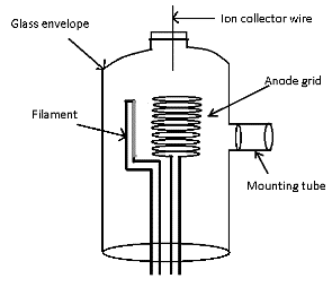

Figure 13 Hot cathode ionization gauge

For high accuracy in using hot cathode ion gauge there is need to control emission current and to measure accurate ion current. The operating ranges for hot cathode ion gauges are $10^{-4}$ to $10^{-11}$ Torr.

\section{APPLICATIONS}

Vacuum supply is an integral part of the planning of new laboratories since it is needed for many different applications. Vacuum aspiration is used to remove gases, debris and spilled liquids by suction. Manual vacuum aspiration is used during endometrial biopsy, uterine evacuation in case of pregnancy

Retrieval Number: 100.1/ijeat.C22520210321

DOI:10.35940/ijeat.C2252.0210321

Journal Website: www.ijeat.org failure and pregnancy termination [17]. The vacuum desiccator is sealable enclosure which requires a pump to remove air or moisture from a desiccation chamber. A vacuum desiccator is used to store hygroscopic dried materials or materials sensitive to oxygen. A vacuum desiccator store fresh food, vegetables, meats, cheese smoked food, coffee, potato chips and dry foods over a long period of time. It limits the growth of aerobic bacteria or fungi and prevent evaporation of volatile component [18-19]. Vacuum frying of potato chips at low temperature and pressure enhances the food quality and reduces the chances of formation of carcinogenic substances. The vacuum cleaner helps in cleaning the computer, keyboard, cobwebs, dust and small piece of papers waste by suction. It removes dust and waste from the place where our hands cannot reach and which is invisible to us. In dry vacuum cleaning, rotary vane pump with a filter mounted in front of the pump are used. Vacuum is used in collecting cells from blood or bone marrow of animals and humans for cell harvesting. Vacuum fractional distillation is used to separate components whose boiling points lie close to one another. For example, fractional vacuum distillation is used to separate fatty acids or other esters. Solid products that are heat sensitive materials such as foods, pharmaceuticals and antibiotics are dried using vacuum drying in shorter drying times and at lower temperature and it retains the integrity of the original items without damaging it with heat. Vacuum dryers involve the use of a reduced pressure atmosphere to surround the product. Microwave vacuum drying of mint leaves shows better results in retaining lightness and greenness as compared to hot air drying [20]. The microwave vacuum drying reduces drying time of mint leaves by $85-90 \%$ compared to hot air drying. The suitability of vacuum dryer for a particular application depends on the solid materials moisture content. The vacuum levels required to filtrate product depends on the thickness and porosity of the filter cake which separates the solids from the liquid. The vacuum requirements for rotatory evaporators depend on the solvent mixtures and evaporating temperature. Therefore, modern vacuum systems include a built-in-vacuum controller to help to achieve the optimum evaporation rates. Rotatory evaporators evaporate liquid by applying heat and reducing the pressure while rotating them in a vessel. The vacuum pump works with a chiller, a vacuum controller and a rotatory evaporator. Tissue culture is the process of keeping tissue in a culture medium alive and growing. Its configuration consists of a vacuum pump, a liquid trap and a pipette. Vacuum is necessary during the production of superconducting thin films and for the thermal insulation of cryostats. In high vacuum evaporation, the electron beam heating is used to melt and evaporate the coating material for deposition onto surface of various substrate forming good films. In high vacuum thin film deposition, only few contaminants are presents to disrupt the growth of thin film.

Published By:

Blue Eyes Intelligence Engineering and Sciences Publication

160 (c) Copyright: All rights reserved.

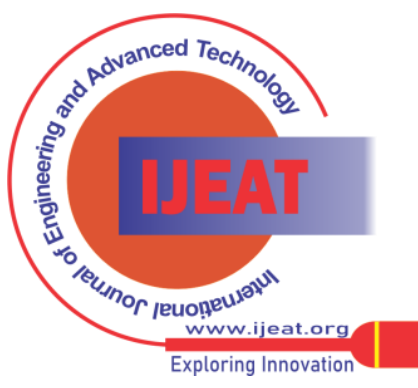


Thin films are kept in vacuum after deposition are not susceptible to oxidation or other reactions due to the absence of gas molecule. Deposition of superconducting thin films using vacuum techniques is an established technology with numerous applications [21]. The large vacuum system at Large Hadron Collider (LHC) at CERN established extremely low pressure in the beam line to avoid collisions during the acceleration of particles. The ion pump has no moving parts with no vibrations so it is well suited for use in scanning probe microscopy and other high precision apparatus. For lifting of loads, the vacuum is created between suction cups and the surface of the load to be displaced. The required vacuum depends on the load weight that will be displaced. High performance thermal insulation is increasingly required to reduce energy consumption. A superior thermal insulation are achieved by vacuum insulation. Vacuum is used in brake systems of automobiles to ensure safe driving. A vacuum pump is used to reduce the time to attain low enough vacuum pressure after the brake loses the vacuum inside the brake booster. A complete simulation of the fabrication, structure design and function circuit of nanoscale vacuum channel transistor has been developed. Nanoscale vacuum channel transistors will enable future on-chip vacuum transistors applied in logic or radio frequency devices [22]. Vacuum technology enabled the design and production of innovative drug delivery systems based on microfabrication of microneedles [23]. The presence of vacuum cavities is useful in reducing noise from fan duct, passenger cabin and other areas of aircraft [24].

\section{RESULTS AND DISCUSSION}

Multiple pumps are connected in series to achieve higher vacuums. The combination of a backing pump and a vacuum booster considerably increases the output and efficiency. The combination of root pump with rotary pump are used to achieve middle vacuum. The ultrahigh vacuum is achieved by using both turbomolecular pump and ion pump. To achieve the ultimate pressure regime of $10^{-11}$ Torr a large speed titanium sublimation pumps are generally used in combination with a sputter ion pump, but this combination is not always feasible due to large size. The compact NEXTorr D2000-10 combination pump is applicable where large load of gases is generated and no more space is available [8]. The pumping speed of oil diffusion pump and turbomolecular pump reaches maximum at $\sim 10^{-3}$ Torr and remain constant up to $10^{-6}$ Torr. The ion pump should not be turned on until vacuum is in the $10^{-4}$ Torr range $\left(\sim 10^{15}\right.$ molecule $\left./ \mathrm{cm}^{3}\right)$. The pumping speed of ion pump reaches maximum at $10^{-6}$ Torr $\left(\sim 10^{11}\right.$ molecule $\left./ \mathrm{cm}^{3}\right)$. So, before selection of vacuum pump, it is important to consider the requirement of degree of vacuum and pumping speed to meet the requirements. The materials used for vacuum system must have sufficient mechanical strength, chemical and corrosion resistant, low intrinsic vapor pressure, high melting and boiling point and have favorable outgassing properties. Some materials which have high vapor pressure like zinc, cadmium, plastics, mercury, magnesium, calcium, sulphur, phosphorus, lead, barium should be avoided for vacuum applications. The gold and silver having low vapor pressure is found to be suitable material for ultrahigh vacuum.
For low outgassing materials like aluminum and stainless steel are generally used. However, titanium, tungsten, tantalum or molybdenum is other option but for specialized application. $\mathrm{Al}_{2} \mathrm{O}_{3}$ ceramics are used in transmitting tubes of vacuum system. Elastomers, thermoplastics, duroplastics and Indium are used for sealing purposes in vacuum systems. The pressure rise is produced by outgassing and gas molecules penetrating through leaks from outside into the vacuum system. Vacuum chambers leaks can affect product quality and damage internal components. The helium leak detection [16] is the ideal solution to determine the vacuum tightness of a large vacuum chamber. It has ability to quickly pinpoint the location and relative magnitude of the leak. It is necessary to clean vacuum vessels and its components in order to reach the desired pressure as quickly as possible when they are pumped down. It is important to determine the level of contamination that can be tolerated in any given process. The hydrocarbon contamination should be reduced to a minimum which are generally achieved by washing in a hot organic solvent like trichloroethylene followed by washing in hot clean demineralized water and the baking of vacuum components usually done at $250^{\circ} \mathrm{C}$ in air to reduce outgassing rates. Electropolishing reduces outgassing by reducing surface area and also dc glow discharge cleaning reduces outgassing and desorption rates. The desorption is induced by energetic particle bombarding the vacuum chamber walls. This induced desorption is dominant source of gas in a vacuum system [15]. A gasket and flange type standard leak element could be used to reduce outgassing and constraints of low baking temperature and are used in gauge calibration and measurement of pumping speeds [25]. The hysteresis in the reading has been observed in measurement by mechanical gauge. The accuracy of thermal conductivity gauge depends on thermal characteristics of gas mixture and the position of its installation. The inaccuracy may arise due to mixture of unknown gases. Therefore, calibration to known standard is required. The calibration of ionization gauge using the comparison method are recommended where ionization gauge pressure reading is compared to a working reference standard [26]. The quantum based standard measuring devices are also recommended since it never needs to be calibrated. Numerical simulation predicts the optimal design of the structural parameters and performance of the internal working process of the pumps such as vacuum chamber volumes, pipe conductance, outgassing from walls, leakage and flow behavior. Computer programs are available for vacuum system simulations for specific duties such as load lock pumping or processing with hydrogen flows. Rapid advanced simulation tools enabled optimization of future large vacuum facilities and complex vacuum electronic circuits. Mathematical modelling of different chamber profile of the vacuum pump has been developed to evaluate the evacuation time which shows better performance of pump with an elliptical chamber and also the evacuation time of the pump decreases for higher value of eccentricity [27].

Published By:

Blue Eyes Intelligence Engineering and Sciences Publication

(C) Copyright: All rights reserved.

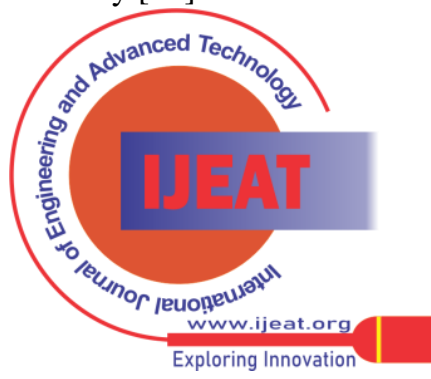




\section{Production, Measurement and Applications of Vacuum Systems}

\section{CONCLUSIONS}

There is no universal vacuum pump and gauge that will work throughout the range from atmospheric pressure to $10^{-13}$ Torr. It is still a challenge to make a reliable gauge for measurements in the extremely high vacuum range. Modeling enhances the functional capability of vacuum systems. The pressure transducer, wireless pressure gauge and digital processing of measurement system with high resolution and accuracy has been developed. There is a need of research on further improving the quality and feasibility of vacuum system as the cost of equipment is too high.

\section{REFERENCES}

1. Erenkov, O Y. Yavorskii, D O. \& Kalenskii, A M. Improving the Efficiency of a Guarded-Vane Vacuum Pump.2020; Chemical and Petroleum Engineering. 55, 1003-1006. https://doi.org/10.1007/s10556020-00727-6;

2. Hong, S., Son, G. Numerical study of a vane vacuum pump with twophase flows. 2017; Journal of Mechanical Science and Technology. 31, 3329-3335. https://doi.org/10.1007/s12206-017-0623-1;

3. Lee, SH., Jeong, HY. A study on the development of a numerical model for a rotary vane vacuum pump for brake boosters. 2018; Journal of Mechanical Science and Technology 32, 3677-3685 https://doi.org/10.1007/s12206-018-0720-9;

4. Lee, S H. Jeong, H Y. Study on the driving torque and target pressure time of a rotary vane pump for brake booster.2017; Journal of Mechanical Science and Technology 31, 5863-5869 https://doi.org/10.1007/s12206-017-1129-6;

5. Hutterer Markus and Schrodl Manfred. Control of active magnetic bearings of turbomolecular pumps for rotors with low resonance frequencies of blade wheel. 2017; Lubricants.5(3), 26. https://doi.org/10.3390/lubricants5030026

6. Fomin, M.V., Chernyshev, O.R. Gas Flow in a Multistage Turbomolecular Vacuum Pump. Russian Engineering Research. 40, 564-566 (2020). https://doi.org/10.3103/S1068798X20070138;

7. Gordeeva U S. Demikhov K E. Ochkov A A. Development of a calculation method of the main parameters of the multistage turbomolecular pump. 2020; IOP Conf. series: Material Science and Engineering,781,012010. doi:10.1088/1757-899X/781/1/012010

8. Luo C. Li P. Xie W.J. Wan Y.P. Chai Z. Zhu X R. Meng J. Chen S P. Yang W S. Application of compact NEG-sputter ion pump combination in UHV system of HIAF.Vacuum.2018;159165.https://doi.org/10.1016/j.vacuum.2018.08.038

9. Verma R. Nagendra H N. Vivek G A. Srinivasan. Kasthurirengan, Shivaprakash N C. Upendra B. Studies on activated carbons towards the performance improvement of cryosorption pump. Cryogenics.2020;108, 103089. https://doi.org/10.1016/j.cryogenics.2020.103089

10. E Ismaiel. Saleh F. Alaaraj B. Barakat N. Material types and wall thickness effects on Bourdon tube tip travel using solid works. 2020; International Journal of Recent Technology and Engineering. 8(5) 4378-4381. Doi:10.35940/ijrte. E6942.018520

11. Wang X. Liu C. Zhang Z. Liu S. Luo X. A micromachined Pirani or vacuum measurement of ultra-small sized vacuum packaging. 2010; Sensors and Actuators A: Physical .161(1-2), 108-113. https://www.doi.org/10.1016/j.sna.2010.04.034

12. Celso de Araujo Duarte. A thermocouple vacuum gauge for low vacuum $\begin{array}{llll}\text { measurement. 2011; } & \text { Vacuum, 85(10), 972-974. }\end{array}$ https://doi.org/10.1016/j.vacuum.2011.02.004

13. B.R.F. Kendall, E. Drubetsky. Compact wide range cold cathode gauges. 2000; Journal of Vacuum Science \& Technology A. 18,1724. https://doi.org/10.1116/1.582479

14. Jousten K. Boineau F. Bundaleski N. Illgen C. Setina J. Orlando M.N.D. Teodoro. Vicar M. Wuest M. A review on hot cathode ionization gauges with focus on a suitable design for measurement accuracy and stability. 2020; Vacuum. 179,109545. https://doi.org/10.1016/j.vacuum.2020.109545

15. Malyshev O B. and Naran C. Electron stimulated desorption from stainless steel at temperature between -15 and $+70^{\circ} \mathrm{C}$. 2012; Vacuum. 6(9), 1363-1366. https://doi.org/10.1016/j.vacuum.2012.01.002
16. Mc Menamin Ed. Helium leak testing: new automotive safety and functionality requirements drive helium leak detection growth. Quality,56(4), April 2017, p-A11+

17. Islam R, Biswas S, Halder D \&, Fatima K. Safety \& efficacy of manual vacuum aspiration compared to dilatation \& curettage in the management of early pregnancy failure.2017; Bangladesh Medical Journal Khulna, 49(1-2),18-22. https://doi.org/10.3329/bmjk.v49i1$\underline{2.31820}$

18. Sun D W, Zheng, L. Vacuum cooling technology for the agri-food industry: Past, present and future. 2006; Journal of Food Engineering,77(2), 203-214

19. Diamante, L M, Shi S, Hellmann A and Busch J. Vacuum frying foods: products process and optimization. 2015; International Food Research Journal.22(1), 15-22

20. Therdthai N, Zhou W. Characterization of microwave vacuum drying and hot air drying of mint leaves (Mentha Cordifolia Opiz ex Fresen). 2009; Journal of Food Engineering,91(3),482-489. https://doi.org/10.1016/j.jfoodeng.2008.09.031

21. Shrivastava Shailaj Kumar. Deposition techniques for high- $T_{c}$ superconducting YBCO thin films. 2017; International Journal of Engineering and Scientific Research. 5(6), 33-43

22. Xu Ji. Qin Yaling. Shi Yongjiao. Yutong Shi, Yang Yang and Zhang Xiaobing. Design and circuit simulation of nanoscale vacuum channel transistors. 2020; Nanoscale Advances.2(8),3582-3587. doi: 10.1039/d0na00442a

23. Qi Lei Wang, Dan Dan Zhu,Yang Chen, Xin Dong Guo, A fabrication method of microneedle molds with controlled microstructures. 2016; Material Science and Engineering C.65.135-142

24. Sandu C. Deaconu M. Silivestru V. Filipescu B. Sandu R C. Noise reduction system in the fan duct and aircraft cabin through vacuuming. The $21^{\text {st }}$ CEAS-ASC Aeroacoustics Workshop, September 2019, Dublin, Ireland.

25. Chan C K. Yeh S D. Chang C C. Tu C Y. Yang I C. Chang K L. Luo C.W. Hwang C S. A flange-type standard leak element and its vacuum applications. 2021; Vacuum, 184,109945. http://doi.org/10.1016/j.vacuum.2020,109945

26. Fedchak J A. Abbott P J. Hendricks J H. Arnold P C. and Peacock N. Recommended practice for calibrating vacuum gauges of the ionization type.2018; Journal of Vacuum Science \& Technology A. 36,030802.https://doi.org/10.1116/1.5025060.

27. Anisha Thaslim A, Suresh Kumar J, Jaychandran A, Damodaran D, and Vasudevan R. Design and analysis of an automotive vacuum pump. Applied Mechanics and Materials 2014, 592-594,18591863.doi:10.4028/www.scientific.net/AMM.592-594.1859

\section{AUTHOR PROFILE}

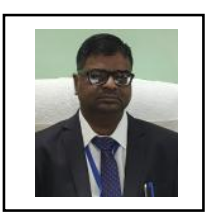

Shailaj Kumar Shrivastava, worked as Research fellow at National Physical Laboratory, New Delhi and obtained his Ph.D. degree in Physical Science from Delhi University in 2002. His research interest is directed towards superconductivity, thin films and devices. Currently he is Principal at Daudnagar College, Aurangabad, Bihar (A constituent unit of Magadh University, Bodh Gaya). He has 25 years of teaching and research experience with more than fifty research papers publications in various Journals and conferences. He got several awards including 'Young Research Award' at IUMRS-ICA-98 held at IISc Bangalore.

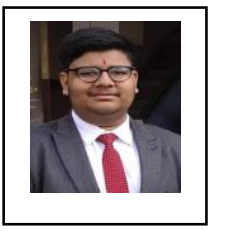

Chandan Shrivastava, is a under graduate student of computer science engineering department at International institute of Information technology, Hyderabad. His interest is directed towards engineering science and technology.

Published By:

Blue Eyes Intelligence Engineering and Sciences Publication

Journal Website: www.ijeat.org

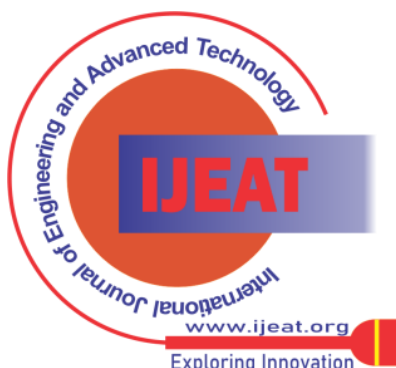

